\title{
ВЛИЯНИЕ ГРАФИКА РАБОТЫ НА СТЕПЕНЬ ВЫРАЖЕННОСТИ ДИАБЕТИЧЕСКОЙ НЕФРОПАТИИ
}

\author{
Гариева М.А., Косян А.А., Аметов А.С., Кондратьева Л.В., Пашкова Е.Ю.
}

ФГБОУ ДПО «Российская медицинская академия непрерывного профессионального образования», Москва

ЦЕЛЬ: оценить Влияние ночного графика работы на развитие диабетической нефропатии у пациентов с сахарным диабетом 2 типа.

MEтоды: в исследовании приняли участие 37 человек с сахарным диабетом 2 типа, находящихся на терапии пероральными сахароснижающими препаратами, в возрасте 35-65 лет.

В первую группу (№1) были включены 22 человека с дневным графиком работы, а во вторую группу (№2) - 15 человек со стажем ночного графика работы более 5 лет в качестве модели пациентов с нарушенным циркадным ритмом. Был проведен сравнительный анализ результатов между обеими группами.

PЕЗУЛЬтАТЫ: проявления диабетической нефропатии исследуемых пациентов были оценены с помощью определения уровня креатинина в крови и расчета CKФ по формуле MDRD.

При сравнительном анализе описательных данных отмечается преобладание уровня креатинина $(p<0,05)$ и соответственно, снижение уровня СКФ $(p>0,05)$ в группе пациентов с ночным графиком работы.

Рис. №1. Оценка функции почек в группах сравнения.

Как известно, кроме плохого гликемического профиля, неблагоприятными прогностическими факторами в развития диабетических осложнений являются возраст пациента, длительность СД2 и АГ, которые за счет совместного влияния на метаболические и гемодинамические процессы внутри почечных клубочков, приводят к прогрессированию диабетической нефропатии. Но, несмотря на сравнительно высокий уровень показателей углеводного обмена, больший стаж СД2, АГ и больший возраст в группе пациентов, работающих днем (p<0,05) (рис. №3), значения СКФ в обеих группах статистически не отличаются и свидетельствуют о снижении фильтрационной способности почек (норма 90-130 кг/м/1,73) в обеих группах (табл. 1). Более раннее снижение фильтрационной способности почек в №2 группе можно объяснить более высоким уровнем ВГ, значение которого имеет прямую корреляционную взаимосвязь с уровнем креатинина $(r=0,656 ; p<0,05)$ и обратную с СКФ $(r=-0,657 ; p<0,05)$.

Таблица 1. Сравнительная характеристика показателей функции почек в группах.

\begin{tabular}{lccc}
\hline \multirow{2}{*}{ Показатели } & Группа №1 $(\mathbf{n = 2 2 )}$ & Группа №2 (n=15) & \multirow{2}{*}{$\mathbf{P}$} \\
\cline { 2 - 3 } & Me;IQR & Me;IQR & \\
\hline Креатинин (мкмоль/л) & 85,$5 ; 14,2$ & 105,$3 ; 21$ & $\mathbf{p = 0 , 0 0 0 2 0 4}$ \\
\hline СКФ по MDRD (кг/м/1,73) & $73 ; 27$ & 64,$5 ; 19$ & $\mathrm{p}=0,163634$ \\
\hline
\end{tabular}

Корреляционный анализ показал, что у пациентов в №2 группе наблюдается положительная корреляция ВГ с весом $(r=0,450 ; p<0,05)$, ИМТ $(r=0,571 ; p<0,05)$, уровнем креатинина $(r=0,656 ; p<0,05)$, баллами

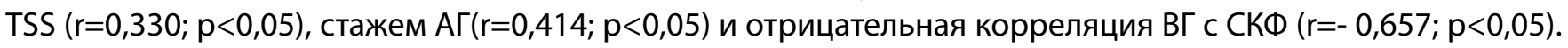
Это означает, что ночной график работы приводит к более раннему прогрессированию диабетических осложнений, за счет значительно выраженной вариабельности гликемии.

ВЫВоды: нарушение циркадного ритма способствует более раннему развитию сахарного диабета и артериальной гипертензии, а также, за счет увеличения вариабельности гликемии и ухудшения эндотелиальной функции, приводит как к более раннему развитию, так и прогрессированию диабетических осложнений, в том числе диабетической нефропатии. 